PACS 63.20.PW

\title{
Large systems of discrete breathers in graphene
}

\author{
J.A. Baimova \\ julia.a.baimova@gmail.com
}

M.N. Miheev Institute of Metal Physics of Ural Branch of RAS (IMP UB RAS), Kovalyevskoy 18, 620990, Ekaterinburg

Nonlinear localized vibrational modes or discrete breathers are of great interest nowadays, because their role in different physical properties is still remaining unknown. Elastic strain engineering allows making quite a wide gap in the density of the phonon states of graphene, although undeformed graphene has no such a gap. Gap discrete breathers in graphene can possibly trigger defect or even crack and fracture formation and the investigation of its properties is of great importance nowadays. Clusters of discrete breathers in graphene can contribute to the considerable energy accumulation and, moreover, the energy exchange between discrete breathers was previously found. At the present work, systems composed of dozens of gap discrete breathers in graphene are investigated by molecular dynamics simulation at zero temperature. Different variations of the initial amplitudes of discrete breathers in the cluster and the combination of two initial phases are considered. It is shown that such clusters can survive for a long time during thousands oscillation periods. "Negative" initial displacement when atoms in the breather are moving towards each other is found to be more preferable in comparison with the "positive" initial displacement when atoms in the breather are moving from each other and allows excitation of the long-living breathers.

Keywords: discrete breathers, molecular dynamics, clusters.

\section{Introduction}

Last decades, intrinsic localized modes or discrete breathers (DB) in a various structures are considered to be one of the most interesting objects in nonlinear science [1-24]. DB were found in one- [1], -two [2,3,4] and three-dimensional $[4,5]$ structures, where they can accumulate quite high amount of energy or even transfer energy to the other lattice objects [6]. To talking about two-dimensional structures, it should be noted, that gap $\mathrm{DB}$ were already found in graphene $[13,14,15,16,17]$. The frequency of such DBs is lying in the wide gap in the density of the phonon states (DOS) $[14,15,16,17]$ or higher than the upper boundary of DOS [13]. Interestingly, gap in the phonon spectrum can be found only for strained graphene $[18,19,20]$.

As it was shown previously, DBs can play a significant role in different physical processes like defect formation, fracture initiation, to name a few. For example, the excitation of the DB in carbon nanotube can trigger the Stone-Wales transformation [21]. Even more structural changes can be triggered by clusters of the DBs.

In the present work, large clusters of DBs in graphene and their properties are investigated by means of molecular dynamics (MD) simulation. Considerable energy exchange between DBs in cluster is shown.

\section{Simulation details}

The initial graphene structure is shown in Fig. 1a. Single discrete breather is initially excited as it is shown in Fig. 1a. All the details of the initial excitation of DB was described previously by author in [17]. Simulation cell contains 16 periodic cells along $x$ and $y$ direction. Periodic cells along $x$ direction have $m$ numbers, which means that each $\mathrm{DB}$ is also having $m$ number. Graphene lattice is subjected to the in-plane strain with the components $\varepsilon_{x x}=0.3, \varepsilon_{y y}=-0.1$ and $\varepsilon_{x y}=0$. The equilibrium positions of atoms in uniformly strained graphene are found by minimizing the potential energy of the crystal. Temperature of the system is equal to $0 \mathrm{~K}$.

All the simulations are carried out with the help of self-written program with the standard set of interatomic potentials $[25,26,27]$. This potential was previously used for studying of DBs in carbon nanostructures and approved to be suitable for such investigations $[15,17,26,27]$.

(a)

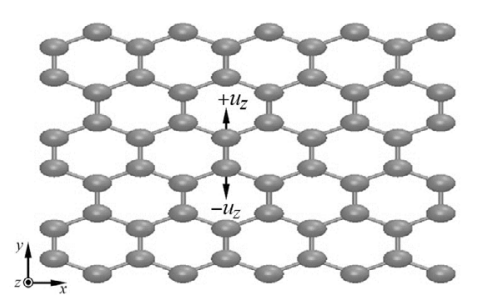

(b)

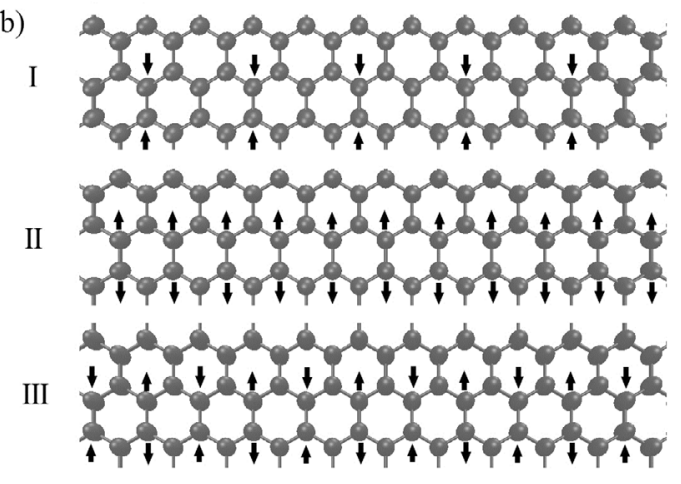

Fig. 1. (a) Graphene structure with single DB and (b) DB systems. 
Clusters of DBs considered in the present work are shown in Fig. 1b. All the three systems are the linear chains of DBs. In the system I DBs have the same phases and only the even DBs are exited with the "negative" initial displacement (atoms are moving towards each other); in the system II - all the DBs (even and odd) have the same "positive" phase (atoms are moving from each other); and in the system III - DBs (even and odd) are excited with the opposite phases alternately. Differences between cases II and III is the phase difference for the even and odd DBs. Directions of the initial movement are shown in Fig. 1 b by arrows. The initial amplitude of DB is also very important, because the difference in the initial amplitudes can lead to the considerable energy exchange between DBs [15]. In the present work, different combinations of the initial amplitudes are considered.

\section{Results and discussion}

In Fig. 2a, the example of the energy exchange in the system is shown for the case I where black curve is for DBs with $m$ numbers and red dashed curve is for DBs with $(m+2)$ numbers. For chosen difference in the initial amplitudes (about two times), considerable energy exchange can be found. From the stroboscopic picture (Fig. 2b) it can be seen that when $m$ DBs are moving with the amplitude $\mathrm{A}_{1},(m+2)$ DBs are moving with the amplitude $A_{2}\left(A_{1}>A_{2}\right)$. Atoms displacements in the stroboscopic picture are enlarged by 10 times.

Various scenario of DBs behavior are shown in Fig. 3. For the case I, with the initial amplitudes of $m$ DBs three times lover the amplitudes of $(m+2)$ DBs, considerable energy exchange can be found for quite long time. For the case III,

(a)

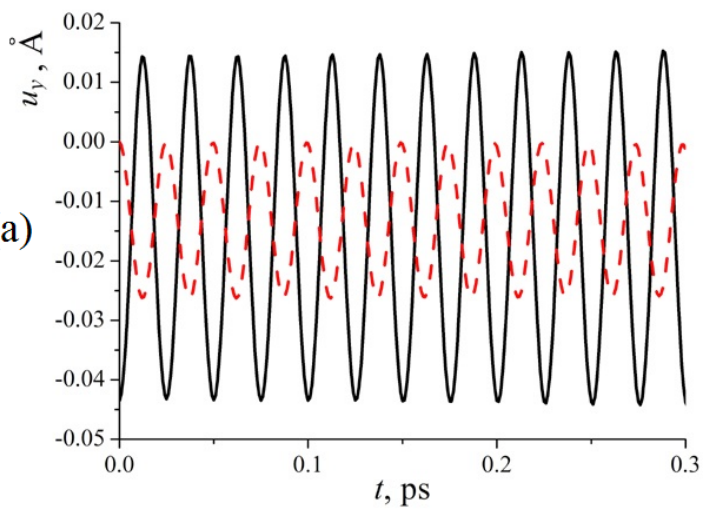

(b)

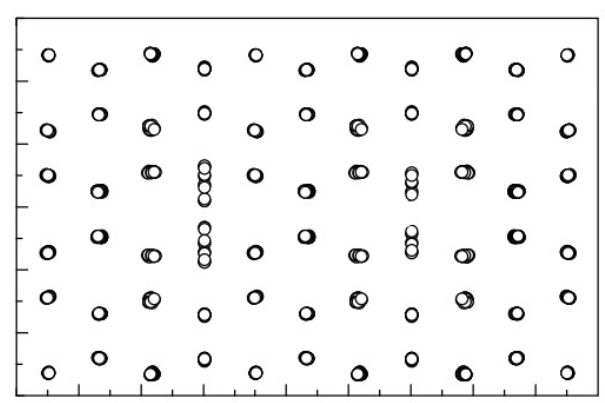

Fig. 2. (color online) (a) Displacement along $y$ direction for one atom of DB as the function of simulation time. Black (red dashed) curve for even (odd) numbers of atoms along $x$ direction. (b) Stroboscopic picture.
DBs excited with the "negative" initial displacements are survive in comparison with DBs excited with the positive initial displacement. This means that the "negative" mode is more preferable than "positive" initial displacement. In this case, odd breathers are gradually dying while even DBs are living for a long time with almost the same amplitude of the oscillations. The same scenario is found for the case II, where DBs are excited with the "positive" mode. This DB system cannot survive for a long time and DBs are dying after about 2 ps, which is quite a long time for $\mathrm{DB}$, but small living time in comparison with the case I.

\section{Conclusions}

In present work, large systems of DBs are investigated by molecular dynamics simulation. Several preferable oscillation modes are observed. "Negative" initial displacement is found to be more preferable in comparison with the "positive" initial displacement and allows exciting long-living breathers. Considerable energy exchange is found for clusters with the initial differences in the breathers amplitudes.

Results presented in this work show that graphene is of great interest in terms of the investigation of gap discrete breathers and its clusters. Energy exchange between DBs observed in the present work is of high importance for the understanding of energy transfer in graphene.

Acknowledgements. Author gratefully thanks grant of Russian Foundation for Basic Research №16-32-00483-мол_a. The simulations were partly carried out on the supercomputer of RAS Supercomputer Center.
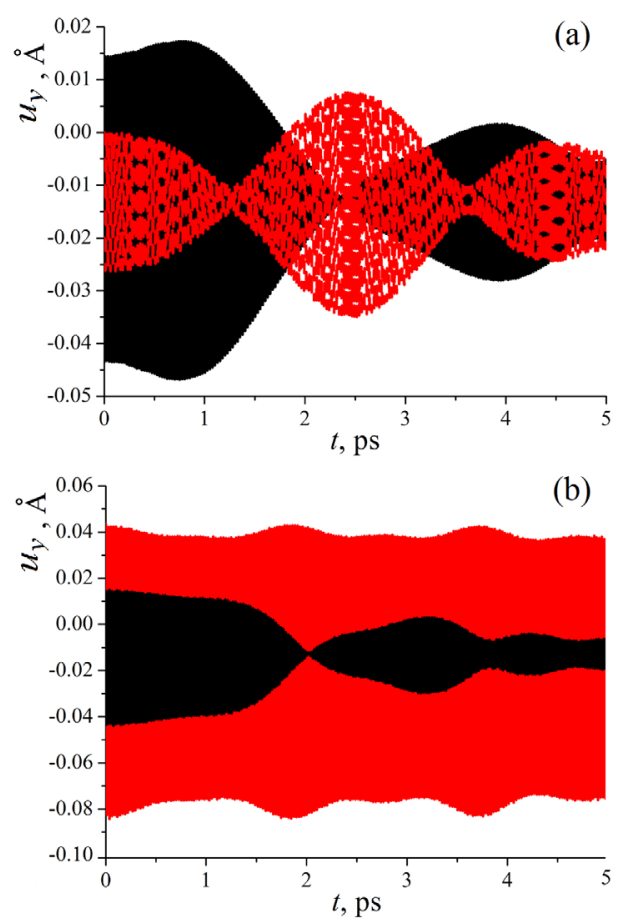

Fig. 3. (color online) Displacement along $y$ direction for one atom of $\mathrm{DB}$ as the function of simulation time: (a) I and (b) III. Black (red dashed) curve - for even (odd) numbers of atoms along $x$ direction. 


\section{References}

1. M. Sato and A. J. Sievers, Phys. Rev. B. 71, 214306 (2005).

2. A.A. Kistanov, S. V. Dmitriev, A.P. Chetverikov and M. G. Velarde. Eur. Phys. J. B. 87, 211 (2014).

3. S. V. Dmitriev, L.Z. Khadeeva, A.I. Pshenichnyuk and N. N. Medvedev. Phys. Solid State. 52 (7), 1499 (2010).

4. S. V. Dmitriev, A.A. Kistanov, V.I. Dubinko. Springer Series in Materials Science. 221, 205 (2015).

5. L.Z. Khadeeva and S.V. Dmitriev. Phys. Rev. B. 81, 214306 (2010).

6. G. Kopidakis and S. Aubry. Physica D. 130, 155 (1999).

7. R. T. Murzaev, A. A. Kistanov, S. V. Dmitriev, V. I. Dubinko, D. A. Terentyev. Comp. Mater. Sci. 98, 88 (2015).

8. J. A. Baimova, S. V. Dmitriev. Russ. Phys. J. 58 (6), 42 (2015).

9. A.S. Semenov, R.T. Murzaev, A.A. Kistanov, Y.V. Bebihov. Fundamentalnie problem sovremennogo materialovedeniya. 12 (1), 26 (2015).

10. J. A. Baimova, S. V. Dmitriev, A. A. Kistanov, A. I. Potekaev. Russ. Phys. J. 56 (2), 180 (2013).

11. S. V. Dmitriev, A.P. Chetverikov, M.G. Velarde. Phys. Stat. Solidi B. 252, 1682 (2015).

12. N. N. Medvedev et al. Russ. Phys. J. 57 (3), 387 (2014).

13. Y. Yamayose, Y. Kinoshita, Y. Doi, A. Nakatani and T. Kitamura. Europhys. Lett. 80, 40008 (2007).
14. E. A. Korznikova, J.A. Baimova and S.V. Dmitriev. Europhys. Lett. 102, 60004 (2013).

15. J. A. Baimova, S. V. Dmitriev and K. Zhou. Europhys. Lett. 100, 36005 (2012).

16. E. A. Korznikova, Y.A. Baimova, S.V. Dmitriev, R. R. Mulyukov and A. V. Savin. JETP Lett. 96, 222 (2012).

17. L.Z. Khadeeva, S. V. Dmitriev and Yu. S. Kivshar. JETP Lett. 94, 539 (2011).

18. Y.A. Baimova, S. V. Dmitriev, A. V. Savin, Y.S. Kivshar. Physics of the Solid State. 54 (4), 866 (2012).

19. J.A. Baimova, S. V. Dmitriev, K. Zhou A. V. Savin. Phys. Rev. B. 86 (3), 035427 (2012).

20. S. V. Dmitriev, Y. A. Baimova. Technical Physics Letters. 37 (5), 451 (2011).

21. T. Shimada, D. Shirasaki and T. Kitamura. Phys. Rev. B. 81, 035401 (2010).

22. A.S. Semenov, E. A. Korznikova, S. V. Dmitriev. Letters on Materials. 5 (1), $11-14$ (2015).

23. A. Kistanov, E. Korznikova, S. Fomin, K. Zhou, S. Dmitriev Letters on materials 4 (2014) 315-318

24. S. V. Dmitriev. Letters on Materials. 1 (2), 78 (2011). (in Russian)

25. A. V. Savin, Yu. S. Kivshar and B.Hu. Phys. Rev. B. 82, 195422 (2010).

26. A. V. Savin and Yu. S. Kivshar. EPL. 89, 46001 (2010).

27. A. V. Savin and Yu. S. Kivshar. Phys. Rev. B. 81, 165418 (2010). 